OPEN ACCESS

Edited by:

Kenneth Shindler,

University of Pennsylvania,

United States

Reviewed by:

Zoe Rebecca Williams,

University of Rochester, United States

Márta Janáky,

University of Szeged, Hungary

*Correspondence:

Kyoung Sook Jeong

bandyoem@naver.com

Specialty section: This article was submitted to Neuro-Ophthalmology, a section of the journal Frontiers in Neurology

Received: 10 August 2019 Accepted: 24 September 2019 Published: 10 October 2019

Citation:

Kim N-H, Kim HJ, Park C-Y and Jeong KS (2019) Retinal Degeneration

After First-Ever Optic Neuritis Helps

Differentiate Multiple Sclerosis and Neuromyelitis Optica Spectrum

Disorder. Front. Neurol. 10:1076.

doi: 10.3389/fneur.2019.01076

\section{Retinal Degeneration After First-Ever Optic Neuritis Helps Differentiate Multiple Sclerosis and Neuromyelitis Optica Spectrum Disorder}

\author{
Nam-Hee Kim ${ }^{1}$, Ho Jin Kim ${ }^{2}$, Cheol-Yong Park ${ }^{3}$ and Kyoung Sook Jeong ${ }^{4 *}$ \\ ${ }^{1}$ Department of Neurology, Dongguk University Ilsan Hospital and Dongguk University-Seoul, Graduate School of Medicine, \\ Goyang, South Korea, ${ }^{2}$ Department of Neurology, National Cancer Center, Goyang, South Korea, ${ }^{3}$ Department of \\ Ophthalmology, Dongguk University Ilsan Hospital and Dongguk University-Seoul, Graduate School of Medicine, Goyang, \\ South Korea, ${ }^{4}$ Department of Occupational and Environmental Medicine, Hallym University Sacred Heart Hospital, Anyang, \\ South Korea
}

Objective: Differentiation between neuromyelitis optica spectrum disorder (NMOSD) and multiple sclerosis (MS) in the early phase is challenging but crucial for treatment and prognosis.

Methods: We performed a prospective cross-sectional study to discriminate NMOSD from MS by evaluating retinal degeneration in optical coherence tomography (OCT) after a first-ever optic neuritis (ON) episode. Seventy-three NMOSD patients and 38 MS patients with $\mathrm{ON}$ at least 3 months prior were assessed by OCT, best-corrected visual acuity (VA), and 2.5\% low-contrast VA. Multivariate linear regression models were used for comparisons. Receiver operating characteristic curves and Youden index were used for determining the discriminative value of retinal nerve fiber layer thickness (RNFL) and VA in distinguishing NMOSD from MS.

Results: Among eyes with retinal degeneration after a first-ever $\mathrm{ON}$ episode $(n=93)$, NMOSD eyes $(n=60)$ presented thinner RNFL $(p<0.001)$ and worsened VA $(p<0.001)$ relative to MS eyes $(n=33)$. Furthermore, a RNFL thinner than $78.9 \mu \mathrm{m}$ had a specificity of $93.9 \%$ for NMOSD; combined with a VA of $<0.4$ decimal, these characteristics provided $100 \%$ specificity for NMOSD.

Conclusions: The first-ever $\mathrm{ON}$ eyes showed more severe retina degeneration in patients with NMOSD than MS, which could establish a cut-off of RNFL thickness and VA to distinguish NMOSD from MS in the early phase.

Keywords: optical coherence tomography, neuromyelitis optica spectrum disorder, multiple sclerosis, optic neuritis, retinal degeneration

\section{INTRODUCTION}

The early and accurate distinction of neuromyelitis optica spectrum disorders (NMOSD) from multiple sclerosis (MS) is important, as the prognosis and treatments for these two diseases are very different $(1,2)$. Although testing for the aquaporin- 4 antibody has facilitated this differentiation, an accurate diagnosis remains challenging, particularly among those with seronegative NMOSD (2). 
MS and NMOSD have often been reported to occur with optic neuritis (ON) (2). Axonal injury occurs to a greater extent in NMOSD, whereas the demyelination associated with MS occurs with some preservation of axons (1). Therefore, the visual prognosis is worse in those with $\operatorname{NMOSD}(1,3)$.

Optical coherence tomography (OCT) has been used to measure the thickness of retinal nerve fiber layer (RNFL) and the macular volume (MV) in patients with MS and $\operatorname{NMOSD}(2,4)$. Over the years, several studies have suggested that by using OCT, RNFL, and MV can be used to detect axonal loss in MS and monitor treatment efficacy $(2,5,6)$. The RNFL consists of retinal ganglion cell axons that coalesce to form the optic nerve (7). MV is determined by the number of retinal ganglion cell bodies, photoreceptors, and other cell types (8). A significant decrease in RNFL thickness and MV has been known to occur in the eyes of patients with MS, with and without a history of ON (2). Moreover, these abnormalities were found to correlate with brain atrophy in MS (9).

The literature has shown a significant reduction in RNFL thickness after ON among patients with NMOSD, much more than among those with MS $(2,7)$. Thus, RNFL thickness has been suggested to be useful in discriminating between NMOSD and MS $(7,10)$. However, in patients with a history of multiple ON episodes, the discrimination using RNFL thickness may not be easy. Cumulative damage from repeated attacks on the optic nerve may cause an extreme reduction in RNFL thickness even among patients with MS, to as severe a degree as in NMOSD $(7,11,12)$. Despite the critical problem of discriminating between these two conditions using OCT, previous studies have focused solely on the differences after considering a history of $\mathrm{ON}$ regardless of the number of episodes. Thus, assessing eyes with a single $\mathrm{ON}$ episode can demonstrate disease-specific pathology in the optic nerve, averting changes obscured by multiple episodes of ON.

Therefore, by studying eyes with the first-ever ON episode, we sought to determine whether RNFL thickness could be used as a diagnostic tool to differentiate between NMOSD and MS. If possible, we also hoped to establish a cut-off value of RNFL thickness for distinguishing between NMOSD and MS in the early stage of disease.

\section{MATERIALS AND METHODS \\ Study Population and Clinical Data}

This observational and cross-sectional study was performed according to the tenets of the Declaration of Helsinki, and was approved by the institutional review board of Dongguk University Ilsan Hospital. All subjects provided informed written consent. We recruited patients with NMOSD who were seropositive for the aquaporin- 4 antibody defined by the revised 2006 diagnostic criteria of Wingerchuk (13) and recruited patients with MS who met the 2010 McDonald criteria (14). Subjects with diabetes, a history of ocular injury, glaucoma, or other ophthalmologic disorders were excluded. In addition, patients within 3 months of acute $\mathrm{ON}$ were excluded to minimize the effect of ON-related edema on OCT measurements (15).

\section{Optical Coherence Tomography}

Retinal imaging was performed using a time-domain OCT (Stratus OCT-3) with OCT 4.0 software (Carl Zeiss Meditec Inc., Dublin, CA). RNFL thickness was measured by the "Fast RNFL Thickness" protocol and the MV was obtained using the "Fast Macular Thickness" scan. Scans with signal strength $<7$ or with artifact were excluded from the analysis. For eyes with extremely poor visual function (unable to fixate), OCT scans were acquired with external fixation of the fellow eye. RNFL thickness for the temporal, superior, nasal, and inferior quadrants and the overall mean of these quadrants as well as the total MV were obtained by OCT as we previously described (16). The normal range of mean RNFL is $82-110 \mu \mathrm{m}$.

\section{Visual Function}

Best-corrected visual acuity (BCVA) with $100 \%$ contrast (high contrast visual acuity, HCVA) was measured using the standard Snellen chart. Sloan letter charts of $2.5 \%$ contrast (Precision Vision, La Salle, IL) were used for low-contrast visual acuity (LCVA). Visual acuity (VA) was expressed using a decimal scale but was transformed to the logarithm of the minimum angle of resolution $(\log M A R)$ for statistical analyses.

\section{Statistical Analysis}

Comparisons between groups were performed using the Student $t$-test or Mann-Whitney test considering normality and the properties of the variables. OCT measures and visual functions were compared between the groups using multivariate linear regression models adjusting for age and disease duration. Pearson's and Spearman's rank order correlations were used to assess correlations among visual functions, the expanded disability status scale (EDSS), and OCT measurements. Receiver operating characteristic (ROC) curves were used to determine the discriminative value of OCT and VA in eyes with a first-ever ON episode to distinguish between NMOSD and MS. The cut-off values were calculated using the Youden index as the points with the best sensitivity-specificity balance (17). Statistical significance was defined as $p<0.05$. Statistical analysis was performed using SPSS version 20 (IBM, NY, USA).

\section{RESULTS}

Seventy-three patients with seropositive NMOSD and 38 with relapsing remitting MS were analyzed. Subjects with NMOSD were older and had a higher ratio of female participants, higher EDSS score, longer disease duration, and higher number of $\mathrm{ON}$ episode than MS patients. Patients' demographic and clinical characteristics are shown in Table $\mathbf{1}$.

Eyes were categorized into six groups: MS without ON episodes $(N=38)$, MS with a single ON episode $(N=33)$, MS with multiple ON episodes $(N=5)$, NMOSD without $\mathrm{ON}$ episodes $(N=45)$, NMOSD with a single ON episode $(N=60)$, and NMOSD with multiple ON episodes $(N=41)$ (Table 2). In the multiple $\mathrm{ON}$ episode group, the number of ON episode, age, or disease duration did not differ between patients with NMOSD or MS. Among those with a single ON episode, there was no 
difference in age or disease duration between patients with MS and NMOSD.

Comparisons of OCT and visual function measures between eyes with MS and NMOSD according to the number of ON episodes

RNFLs in affected eyes were thinner in all four quadrants in NMOSD eyes relative to MS eyes $(p<0.001$ for each, Table 2), whereas RNFLs in unaffected eyes did not differ between patients with NMOSD and MS, except for a thinner temporal quadrant in MS eyes $(p=0.009)$. When comparing RNFL thickness among eyes with a single ON episode, the RNFLs on average as well as all quadrants were thinner in NMOSD eyes relative to MS eyes ( $p<0.001$ for each; Table 2, Figure 1). Among eyes from patients with multiple ON episodes, RNFLs on average as well as all quadrants except the temporal

TABLE 1 | Demographic characteristics of patients with neuromyelitis optica spectrum disorders or multiple sclerosis.

\begin{tabular}{lccc}
\hline & $\begin{array}{c}\text { Neuromyelitis optica } \\
\text { spectrum disorder }\end{array}$ & $\begin{array}{c}\text { Multiple } \\
\text { sclerosis }\end{array}$ & p-value \\
\hline Number of subjects & 73 & 38 & \\
Mean age, years (SD) & $39.4(12.0)$ & $35.2(10.0)$ & 0.026 \\
Gender, F:M & $63: 10$ & $24: 14$ & $<0.001$ \\
Median EDSS (ranges) & $3.5(1.0-9.0)$ & $1.5(0-7.5)$ & $<0.001$ \\
$\begin{array}{l}\text { Mean disease duration, } \\
\text { years (SD) }\end{array}$ & $6.5(4.5)$ & $4.5(3.8)$ & $<0.001$ \\
$\begin{array}{l}\text { Number of optic nerves } \\
\text { affected by ON (\%) }\end{array}$ & $101(69)$ & $38(50)$ & 0.003 \\
$\begin{array}{l}\text { Median episodes of ON } \\
\text { (ranges) }\end{array}$ & $1.0(0-12)$ & $0(0-11)$ & 0.003 \\
NMO-lgG positive (\%) & 100 & 0 & $<0.001$ \\
\hline
\end{tabular}

MS, multiple sclerosis; NMO, neuromyelitis optica spectrum disorder; ON, optic neuritis; EDSS, Expanded Disability Status Scale. quadrant were thinner in NMOSD eyes than in MS eyes $(p<$ 0.001 for nasal, superior, and inferior quadrants; $p=0.595$ for the temporal quadrant; see Table 2). In addition, the thickness of RNFLs in NMOSD eyes with a single ON episode did not differ from those of MS eyes with multiple ON episodes, suggesting the importance of controlling for the number of ON episodes when comparing eyes of these different diseases (Table 2).

The total MV in affected eyes of NMOSD was reduced compared to those with MS $(p<0.001$; Table 2$)$, whereas there

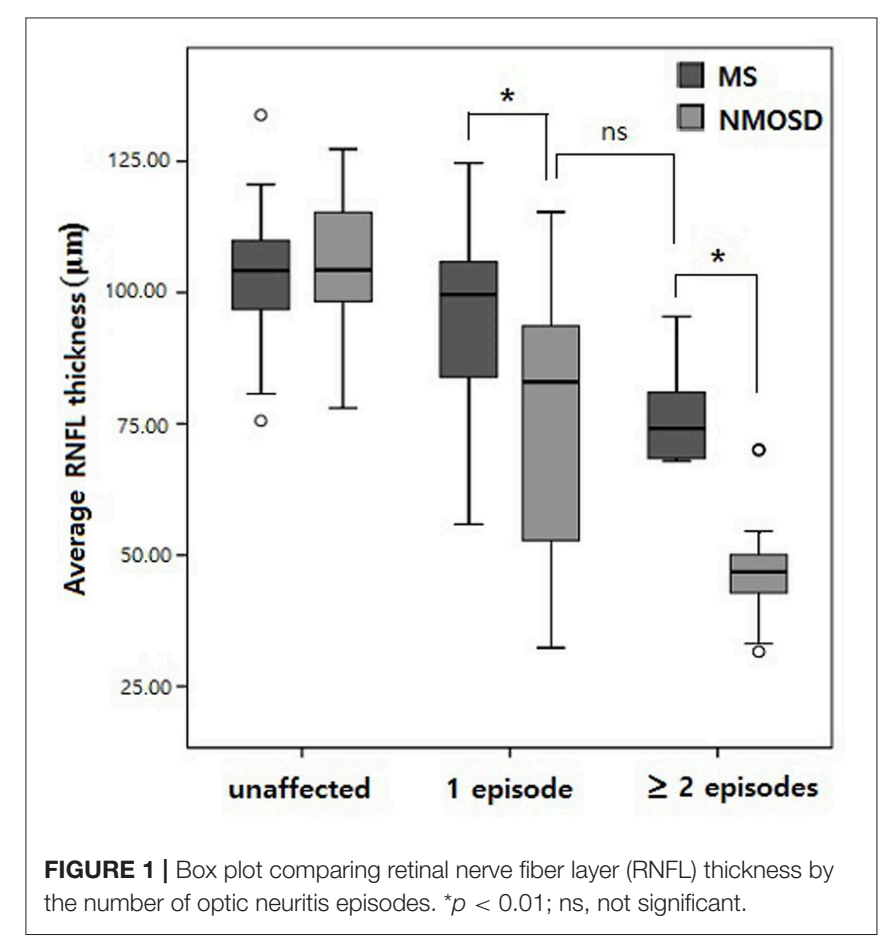

TABLE 2 | Retinal nerve fiber thicknesses and visual functions for multiple sclerosis and neuromyelitis optica spectrum disorder.

\begin{tabular}{|c|c|c|c|c|c|c|c|}
\hline & \multicolumn{3}{|c|}{ Neuromyelitis optica spectrum disorder } & \multicolumn{3}{|c|}{ Multiple sclerosis } & \multirow{3}{*}{$\begin{array}{c}\text { p-value } \\
\text { Single ON } \\
\text { NMOSD vs. MS }\end{array}$} \\
\hline & \multirow{2}{*}{$\begin{array}{l}\text { Unaffected } \\
\qquad(N=45)\end{array}$} & \multicolumn{2}{|c|}{ Affected } & \multirow{2}{*}{$\begin{array}{l}\text { Unaffected } \\
\qquad(N=38)\end{array}$} & \multicolumn{2}{|c|}{ Affected } & \\
\hline & & $\begin{array}{l}\text { Single ON } \\
(N=60)\end{array}$ & $\begin{array}{l}\text { Multiple ON } \\
\qquad(N=41)\end{array}$ & & $\begin{array}{c}\text { Single ON } \\
(N=33)\end{array}$ & $\begin{array}{l}\text { Multiple ON } \\
\qquad(N=5)\end{array}$ & \\
\hline \multicolumn{8}{|c|}{ RNFL thickness, $\mu \mathrm{m}$, mean (SD) } \\
\hline Average & $105.5(11.9)$ & $76.0(24.8)$ & $49.9(16.4)$ & $102.7(11.9)$ & $95.2(14.0)$ & $77.4(11.4)$ & $<0.001$ \\
\hline Temporal & $79.0(15.7)$ & $57.0(21.8)$ & $40.6(14.2)$ & $73.9(17.3)$ & $71.4(24.8)$ & $44.2(12.2)$ & 0.008 \\
\hline Inferior & $137.9(19.3)$ & $98.9(36.3)$ & $58.4(27.1)$ & $133.3(16.3)$ & $123.2(20.8)$ & $99.4(13.2)$ & $<0.001$ \\
\hline Nasal & $78.6(16.3)$ & $56.3(18.1)$ & $43.7(11.2)$ & $78.6(14.6)$ & $68.8(12.4)$ & $65.2(8.9)$ & $<0.001$ \\
\hline Superior & $126.7(18.6)$ & $91.9(34.9)$ & $57.0(21.9)$ & $124.5(17.4)$ & $117.2(18.8)$ & $100.4(20.0)$ & $<0.001$ \\
\hline \multicolumn{8}{|c|}{ Macular volume, $\mathrm{mm}^{3}$, mean (SD) } \\
\hline & $6.77(0.43)$ & $6.07(0.77)$ & $5.50(0.43)$ & $6.75(0.36)$ & $6.58(0.37)$ & $6.27(0.41)$ & $<0.001$ \\
\hline \multicolumn{8}{|c|}{ Visual acuity, LogMAR, median (mean) } \\
\hline $100 \%$ contrast & $0.10(0.26)$ & $0.75(0.93)$ & $1.60(1.63)$ & $0.20(0.35)$ & $0.10(0.33)$ & $1.30(1.10)$ & $<0.001$ \\
\hline $2.5 \%$ contrast & $0.60(0.81)$ & $1.80(1.24)$ & $1.80(1.74)$ & $0.50(0.60)$ & $0.80(1.02)$ & $0.80(1.00)$ & 0.118 \\
\hline
\end{tabular}

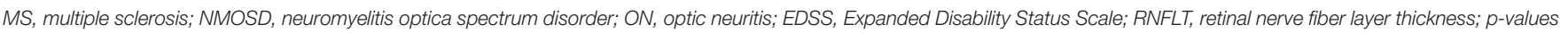
after adjusting age and disease duration. 
was no difference in MV between unaffected eyes of NMOSD and those with MS. In each group categorized with the number of ON episodes, the MV was significantly reduced among eyes with NMOSD than in those with MS ( $p<0.001$ for groups with a single ON episode, $p=0.001$ for groups with multiple ON episodes, Table 2). However, there was no difference in the MV between NMOSD eyes with a single ON episode and MS eyes with multiple episodes of $\mathrm{ON}$.

HCVA and LCVA were worse in the affected eyes of NMOSD compared to those of MS $(p<0.001)$, whereas those were not different between unaffected eyes of NMOSD and unaffected eyes of MS (Table 2). When comparing the eyes with a single ON episode, HCVA was significantly worse in NMOSD relative to MS $(p<0.001)$. LCVA did not differ between NMOSD and MS. HCVA and LCVA in NMOSD eyes with a single ON episode did not differ from MS eyes with multiple episodes of ON.

Among patients with a history of a single unilateral $\mathrm{ON}$, the difference in RNFL thickness between both eyes was significantly larger among patients with NMOSD $(N=10,24.1 \pm 18.8 \mu \mathrm{m})$ relative to patients with $\mathrm{MS}(N=8,10.3 \pm 4.1 \mu \mathrm{m})(p=0.004)$.

Discrimination between eyes with MS and NMOSD after a first-ever ON.

TABLE 3 | Receiver operating characteristic curve analysis of the retinal nerve fiber layer thickness, the macular volume, and visual acuity in those with a first-ever ON episode.

\begin{tabular}{|c|c|c|c|c|c|c|}
\hline & AUC & $\begin{array}{c}p- \\
\text { value }\end{array}$ & $\begin{array}{c}\text { Cut- } \\
\text { off } \\
\text { value }\end{array}$ & $\begin{array}{c}\text { Specificity } \\
\text { for } \\
\text { NMOSD }\end{array}$ & $\begin{array}{l}\text { Sensitivity } \\
\text { for } \\
\text { NMOSD }\end{array}$ & J-index \\
\hline \multicolumn{7}{|c|}{ RNFL thickness, $\mu \mathrm{m}$} \\
\hline Average & 0.704 & 0.001 & 78.9 & 0.94 & 0.45 & 0.39 \\
\hline Temporal & 0.673 & 0.006 & 50.0 & 0.85 & 0.45 & 0.30 \\
\hline Inferior & 0.699 & 0.002 & 123.5 & 0.58 & 0.77 & 0.34 \\
\hline Nasal & 0.717 & 0.001 & 58.5 & 0.81 & 0.53 & 0.35 \\
\hline Superior & 0.734 & $<0.001$ & 101.0 & 0.88 & 0.53 & 0.41 \\
\hline $\mathrm{MV}, \mathrm{mm}^{2}$ & 0.722 & $<0.001$ & 6.3 & 0.85 & 0.45 & 0.37 \\
\hline \multicolumn{7}{|l|}{ VA, decimal } \\
\hline $\begin{array}{l}100 \% \\
\text { contrast }\end{array}$ & 0.704 & 0.001 & 0.4 & 0.73 & 0.65 & 0.38 \\
\hline
\end{tabular}

RNFL, retinal nerve fiber layer; MV, macular volume; VA, visual acuity; LogMAR, Logarithm of the minimum angle of resolution; NMOSD, neuromyelitis optica spectrum disorder; AUC, area under the Receiver operating characteristics curve; J-index, Youden index.
After a first episode of ON, RNFL thickness, MV, and HCVA in NMOSD were significantly worse compared to those of MS ( $p$ $<0.001$; Table 2).

In ROC curve analyses, the average RNFL thickness "cut-off" value was $78.9 \mu \mathrm{m}$ with $93.9 \%$ specificity and $45.0 \%$ sensitivity for discrimination of NMOSD from MS. And HCVA "cut-off" value was 0.40 in LogMAR (0.40 in decimal) with $72.7 \%$ specificity and $65.0 \%$ sensitivity for discrimination of NMOSD from MS. Furthermore, the cut-off value of RNFL thickness $<78.9 \mu \mathrm{m}$ with HCVA $<0.40$ decimal showed $100 \%$ specificity for NMOSD (Table 3).

RNFL thickness in a first-ever ON episode correlated with disability and disease duration.

When evaluating all 93 eyes with a first-ever ON episode, RNFL thickness demonstrated strong negative correlations with HCVA, 2.5\% LCVA, EDSS, and disease duration (Table 4). Among the MS eyes with a first-ever ON episode $(N=33)$, RNFL thickness was significantly correlated with $2.5 \%$ LCVA, EDSS, and disease duration (Table 4). In the analysis of the NMOSD eyes with a first-ever ON episode $(N=60)$, RNFL thickness was significantly correlated with HCVA, 2.5\% LCVA, EDSS, and disease duration (Table 4).

\section{DISCUSSION}

Multiple episodes of ON in the advanced disease state can result in cumulative and extensive axonal damage as well as visual disturbance $(18,19)$ and may thus attenuate differences between NMOSD and MS. Our study confirmed that the NMOSD eyes with a single ON attack showed the thinning of the RNFL and visual disturbances similar to that of MS eyes with multiple ON attacks, supporting the idea that cumulative damage of repeat attacks can mask disease-specific effects on optic nerve. Previous studies using OCT have compared affected or unaffected eyes for both diseases without considering the number of $\mathrm{ON}$ episodes $(2,7,20)$. Thus, a study examining eyes with a first-ever ON episode may be more useful in understanding disease-specific retinal pathology.

We found that after a single episode of ON, thinning of the peripapillary RNFL was significantly greater in the eyes of NMOSD compared with those of MS and was paralleled by

TABLE 4 | Correlations among the RNFL thickness, HCVA, LCVA, EDSS score, and disease duration.

\begin{tabular}{|c|c|c|c|c|}
\hline & HCVA & LCVA & EDSS & Disease duration \\
\hline \multicolumn{5}{|c|}{ All first-ever ON eyes $(N=93)$} \\
\hline RNFLT & $r=-0.643(p<0.001)$ & $\rho=-0.471(p<0.001)$ & $r=-0.426(p<0.001)$ & $r=-0.324(p=0.002)$ \\
\hline \multicolumn{5}{|c|}{ First-ever ON eyes of MS $(N=33)$} \\
\hline RNFLT & $r=-0.034(p=0.852)$ & $\rho=-0.374(p=0.035)$ & $r=-0.376(p=0.031)$ & $r=-0.374(p=0.032)$ \\
\hline \multicolumn{5}{|c|}{ First-ever ON eyes of NMOSD $(N=60)$} \\
\hline
\end{tabular}

r, Pearson's r; $\rho$, Spearman's $\rho$; RNFLT, retinal nerve fiber layer thickness; HCVA, high contrast visual acuity; LCVA, low contrast visual acuity; EDSS, Extended Disability Status Score; ON, optic neuritis; $p$-values are given brackets. 
poorer HCVA. In a systemic review and meta-analysis, the intereye RNFL difference was $30.98 \mu \mathrm{m}$ in NMOSD and $9.87 \mu \mathrm{m}$ in MS between eyes with or without $\mathrm{ON}$, in which $\mathrm{ON}$ included both single and multiple episodes (21). In our study, 29.5 and $7.5 \mu \mathrm{m}$ in RNFL thickness were decreased after single ON attack in NMOSD and MS, respectively. These differences are consistent with findings reported by previous seminal studies using OCT $(2,7,10,20,22)$.

Furthermore, we could calculate cut-off values for discriminating NMOSD from MS among those with a firstever ON episode. We chose cut-off values for RNFL thickness and HCVA excluding MV, because MV is strongly associated with RNFL which consists one of the layers in the macula. Accordingly, we extracted the average cut-off value of $78.9 \mu \mathrm{m}$ for RNFL thickness and 0.4 decimal for HCVA. Using the average RNFL thickness $<78.9 \mu \mathrm{m}$, the specificity was $93.9 \%$ for discriminating NMOSD from MS. RNFL thickness of $<78.9 \mu \mathrm{m}$ with HCVA of $<0.4$ decimal showed $100 \%$ specificity for NMOSD. These results support the theory that RNFL thickness and HCVA could be used as tools for differential diagnosis of MS and NMOSD. In a study by Peng et al. they suggested cut-off value of inferior nasal RNFL thickness in NMO-ON as $\leq 46.5 \mu \mathrm{m}$ for discrimination with healthy control and the specificity was $57.5 \%$ (23). Our study suggests the cut-off value to distinguish between NMOSD and MS after the first-ever ON with an average of $78.9 \mu \mathrm{m}$ for all quadrants of RNFL thickness.

Interestingly, the association of structural retinal damage and impairment of visual function was more apparent in patients with NMOSD relative to patients with MS. In a first-ever ON episode, RNFL thickness and HCVA had a significant correlation among patients with NMOSD $(r=-0.643, p<0.001)$ but not among patients with MS $(r=-0.034, p=0.852)$. Furthermore, a significant correlation between EDSS and RNFL thickness among patients with NMOSD was confirmed in our study with the relatively large cohort, while there has previously been controversy about the association of RNFL thickness and EDSS in this patient population $(2,3,8,11,24,25)$.

We compared the seropositive NMOSD patients with MS patients to avoid controversy over the diagnosis of NMOSD.

\section{REFERENCES}

1. Weinshenker BG. Neuromyelitis optica is distinct from multiple sclerosis. Arch Neurol. (2007) 64:899-901. doi: 10.1001/archneur.64.6.899

2. Bennett JL, de Seze J, Lana-Peixoto M, Palace J, Waldman A, Schippling $\mathrm{S}$, et al. Neuromyelitis optica and multiple sclerosis: seeing differences through optical coherence tomography. Mult Scler. (2015) 21:678-88. doi: $10.1177 / 1352458514567216$

3. Merle H, Olindo S, Donnio A, Richer R, Smadja D, Cabre P. Retinal peripapillary nerve fiber layer thickness in neuromyelitis optica. Invest Ophthal Vis Sci. (2008) 49:4412-7. doi: 10.1167/iovs.08-1815

4. Frohman EM, Fujimoto JG, Frohman TC, Calabresi PA, Cutter G, Balcer LJ. Optical coherence tomography: a window into the mechanisms of multiple sclerosis. Nat Clin Pract Neurol. (2008) 4:664-75. doi: 10.1038/ncpneuro0950

5. Fisher JB, Jacobs DA, Markowitz CE, Galetta SL, Volpe NJ, NanoSchiavi ML, et al. Relation of visual function to retinal nerve fiber layer thickness in multiple sclerosis. Ophthalmology. (2006) 113:324-32. doi: 10.1016/j.ophtha.2005.10.040
However, given the recently published papers, the results of this study may also be applicable to the seronegative NMOSD patients $(26,27)$.

In summary, this study revealed that the RNFL thickness among those with a first-ever ON episode was useful in understanding disease-specific retinal pathology. RNFL thickness and HCVA after a first-ever ON was significantly different among eyes with NMOSD than eyes with MS. We suggest that RNFL thickness with visual function among those with a first-ever ON episode will help discriminate NMOSD from MS in the early stage of diseases, by using cutoff values for the average RNFL thickness and HCVA.

\section{DATA AVAILABILITY STATEMENT}

The datasets generated for this study are available on request to the corresponding author.

\section{ETHICS STATEMENT}

The studies involving human participants were reviewed and approved by Institutional review board of Dongguk University Ilsan Hospital. The patients/participants provided their written informed consent to participate in this study.

\section{AUTHOR CONTRIBUTIONS}

$\mathrm{N}-\mathrm{HK}, \mathrm{HK}$, and C-YP contributed to the design of the work and interpreted the results. N-HK and KJ executed the analyses and wrote the draft of the paper. HK and C-YP supervise the work. $\mathrm{KJ}$ revised the manuscript. All authors read and approved the final manuscript.

\section{FUNDING}

This study was supported by Korea Research Foundation Grant funded by Korea Government (KRF 2009-0067502) to N-HK.
6. Henderson AP, Trip SA, Schlottmann PG, Altmann DR, Garway-Heath DF, Plant GT, et al. An investigation of the retinal nerve fibre layer in progressive multiple sclerosis using optical coherence tomography. Brain. (2008) 131:27787. doi: $10.1093 /$ brain/awm 285

7. Ratchford JN, Quigg ME, Conger A, Frohman T, Frohman E, Balcer LJ, et al. Optical coherence tomography helps differentiate neuromyelitis optica and MS optic neuropathies. Neurology. (2009) 73:302-8. doi: 10.1212/WNL.0b013e3181a f78b8

8. von Glehn F, Jarius S, Cavalcanti Lira RP, Alves Ferreira MC, von Glehn FH, Costa ECSM, et al. Structural brain abnormalities are related to retinal nerve fiber layer thinning and disease duration in neuromyelitis optica spectrum disorders. Mult Scler. (2014) 20:1189-97. doi: 10.1177/1352458513 519838

9. Gordon-Lipkin E, Chodkowski B, Reich DS, Smith SA, Pulicken M, Balcer LJ, et al. Retinal nerve fiber layer is associated with brain atrophy in multiple sclerosis. Neurology. (2007) 69:1603-9. doi: 10.1212/01.wnl.0000295995.46 586.ae 
10. Naismith RT, Tutlam NT, Xu J, Klawiter EC, Shepherd J, Trinkaus K, et al. Optical coherence tomography differs in neuromyelitis optica compared with multiple sclerosis. Neurology. (2009) 72:1077-82. doi: 10.1212/01.wnl.0000345042.53843.d5

11. Lange AP, Sadjadi R, Zhu F, Alkabie S, Costello F, Traboulsee AL. Spectral-domain optical coherence tomography of retinal nerve fiber layer thickness in NMO patients. J Neuroophthalmol. (2013) 33:213-9. doi: 10.1097/WNO.0b013e31829c510e

12. Petzold A, Wattjes MP, Costello F, Flores-Rivera J, Fraser CL, Fujihara K, et al. The investigation of acute optic neuritis: a review and proposed protocol. Nat Rev Neurol. (2014) 10:447-58. doi: 10.1038/nrneurol.2014.108

13. Wingerchuk DM, Lennon VA, Pittock SJ, Lucchinetti CF, Weinshenker BG. Revised diagnostic criteria for neuromyelitis optica. Neurology. (2006) 66:1485-9. doi: 10.1212/01.wnl.0000216139.44259.74

14. Polman CH, Reingold SC, Banwell B, Clanet M, Cohen JA, Filippi M, et al. Diagnostic criteria for multiple sclerosis: 2010 revisions to the McDonald criteria. Ann Neurol. (2011) 69:292-302. doi: 10.1002/ana.22366

15. Henderson AP, Altmann DR, Trip AS, Kallis C, Jones SJ, Schlottmann PG, et al. A serial study of retinal changes following optic neuritis with sample size estimates for acute neuroprotection trials. Brain. (2010) 133:2592-602. doi: 10.1093/brain/awq146

16. Jeong IH, Kim HJ, Kim NH, Jeong KS, Park CY. Subclinical primary retinal pathology in neuromyelitis optica spectrum disorder. J Neurol. (2016) 263:1343-8. doi: 10.1007/s00415-016-8138-8

17. Youden WJ. Index for rating diagnostic tests. Cancer. (1950) 3:32-5. doi: 10.1002/1097-0142(1950)3:1<32::AID-CNCR2820030106>3.0.CO;2-3

18. Bichuetti DB, de Camargo AS, Falcao AB, Goncalves FF, Tavares IM, de Oliveira EM. The retinal nerve fiber layer of patients with neuromyelitis optica and chronic relapsing optic neuritis is more severely damaged than patients with multiple sclerosis. J Neuroophthalmol. (2013) 33:220-4. doi: 10.1097/WNO.0b013e31829f39f1

19. Nakamura M, Nakazawa T, Doi H, Hariya T, Omodaka K, Misu T, et al. Early high-dose intravenous methylprednisolone is effective in preserving retinal nerve fiber layer thickness in patients with neuromyelitis optica. Graefes Arch Clin Exp Ophthalmol. (2010) 248:1777-85. doi: 10.1007/s00417-010-1344-7

20. Noval S, Contreras I, Munoz S, Oreja-Guevara C, Manzano B, Rebolleda G. Optical coherence tomography in multiple sclerosis and neuromyelitis optica: an update. Mult Scler Int. (2011) 2011:472790. doi: 10.1155/2011/472790

21. Peng A, Qiu X, Zhang L, Zhu X, He S, Lai W, et al. Evaluation of the retinal nerve fiber layer in neuromyelitis optica spectrum disorders:
A systematic review and meta-analysis. J Neurol Sci. (2017) 383:108-13. doi: 10.1016/j.jns.2017.10.028

22. Schneider E, Zimmermann H, Oberwahrenbrock T, Kaufhold F, Kadas EM, Petzold A, et al. Optical coherence tomography reveals distinct patterns of retinal damage in neuromyelitis optica and multiple sclerosis. PLoS ONE. (2013) 8:e66151. doi: 10.1371/journal.pone.0066151

23. Peng CX, Li HY, Wang W, Wang JQ, Wang L, Xu QG, et al. Retinal segmented layers with strong aquaporin-4 expression suffered more injuries in neuromyelitis optica spectrum disorders compared with optic neuritis with aquaporin-4 antibody seronegativity detected by optical coherence tomography. Br J Ophthalmol. (2017) 101:1032-7. doi: 10.1136/bjophthalmol-2016-309412

24. de Seze J, Blanc F, Jeanjean L, Zephir H, Labauge P, Bouyon M, et al. Optical coherence tomography in neuromyelitis optica. Arch Neurol. (2008) 65:920-3. doi: 10.1001/archneur.65.7.920

25. Outteryck O, Majed B, Defoort-Dhellemmes S, Vermersch P, Zephir H. A comparative optical coherence tomography study in neuromyelitis optica spectrum disorder and multiple sclerosis. Mult Scler. (2015) 21:1781-93. doi: $10.1177 / 1352458515578888$

26. Bertsch-Gout M, Loeb R, Finch AK, Javed A, Bernard J. High resolution retinal scanning reveals regional structural differences between MS and NMOSD optic neuritis regardless of antibody status. J Neurol Sci. (2018) 384:61-6. doi: 10.1016/j.jns.2017.11.017

27. Martinez-Lapiscina EH, Sepulveda M, Torres-Torres R, Alba-Arbalat S, Llufriu S, Blanco Y, et al. Usefulness of optical coherence tomography to distinguish optic neuritis associated with AQP4 or MOG in neuromyelitis optica spectrum disorders. Ther Adv Neurol Disord. (2016) 9:436-40. doi: $10.1177 / 1756285616655264$

Conflict of Interest: The authors declare that the research was conducted in the absence of any commercial or financial relationships that could be construed as a potential conflict of interest.

Copyright (c) 2019 Kim, Kim, Park and Jeong. This is an open-access article distributed under the terms of the Creative Commons Attribution License (CC BY). The use, distribution or reproduction in other forums is permitted, provided the original author(s) and the copyright owner(s) are credited and that the original publication in this journal is cited, in accordance with accepted academic practice. No use, distribution or reproduction is permitted which does not comply with these terms. 